EPJ Web of Conferences 19, 07004 (2012)

DOI: $10.1051 /$ epjconf/20121907004

(C) Owned by the authors, published by EDP Sciences, 2012

\title{
The collective origin of spiral structures in disk galaxies
}

\author{
E. D’Onghia ${ }^{1,2, a}$, M. Vogelsberger ${ }^{1}$ and L. Hernquist ${ }^{1}$ \\ ${ }^{1}$ Keck fellow \\ ${ }^{2}$ Harvard-Smithsonian Center for Astrophysics, 60 Garden Street, Cambridge, \\ MA 02138, USA
}

\begin{abstract}
After almost fifty years the origin of spiral arms in disk galaxies remains one of the major unsolved problems in astrophysics. Recent attempts have indicated that substructures in the dark matter halos of galaxies could induce spiral patterns in disks by generating localized disturbances that grow by swing amplification. However, there are indications that dark matter substructures orbiting in the inner regions of galaxy halos would be destroyed by dynamical processes such as disk shocking, and hence would not be able to seed the formation of spiral structure. Instead, we use numerical simulations of unprecedented resolution explore the possibility that spiral arms might be generated by the dynamical response of the disk to overdensities corotating within the disk. These perturbations can be identified with fluctuations in the distribution of gas in the interstellar medium of galaxies, such as giant molecular clouds. We develop a new theory for spiral structure formation based on the non-linear effects of swing amplification. Our model makes numerous testable predictions, making it possible to finally confront theory with observations.
\end{abstract}

\section{INTRODUCTION}

Several models have been proposed to explain the formation of spiral arms. The theory proposed by Lin \& Shu (1964) suggests that the arms in galaxies are density waves propagating through a differentially rotating disk. In their picture, the observed spiral pattern is a global wave pattern which either remains stationary or quasi-stationary in a frame of reference rotating around the center of the galaxy at an angular speed equal to the fixed pattern speed of the wave. In order to avoid the winding problem, this theory requires that while the gas and stars rotate differentially in the disk, spiral arms must be uniformly rotating patterns and be long-lived.

An alternative theory proposes that the spiral arms are stochastically produced by the linear gravitational response of a differentially rotating stellar disk to the presence of interstellar material orbiting within its plane (Julian \& Toomre 1966). This mechanism is termed swing amplification and results from the linear response of the disk to any overdensity corotating with the disk that evokes a strong wakelet in the surrounding stellar medium. The wake is amplified by the self-gravity of the disk and amplifies by swinging in a differentially rotating disk (Julian \& Toomre 1966). In this theory, spiral arms are considered to be transient features, unless the perturbers generating the response are permanent.

Most recently, some studies have proposed that the continuous interaction between substructures in the dark matter halos of galaxies and disks could induce spiral patterns by generating localized disturbances that grow by swing amplification (Gauthier et al. 2006). According to the simulations, the main agent producing transient features would be satellite passages through the inner parts of the disk. Because the tidal effects of the satellites are generally small, this process is distinct from interactions thought to be responsible for grand-design spirals like M51. However, there are indications that dark

\footnotetext{
ae-mail: edonghia@cfa.harvard.edu
}

This is an Open Access article distributed under the terms of the Creative Commons Attribution-Noncommercial License 3.0, which permits unrestricted use, distribution, and reproduction in any noncommercial medium, provided the original work is properly cited. 

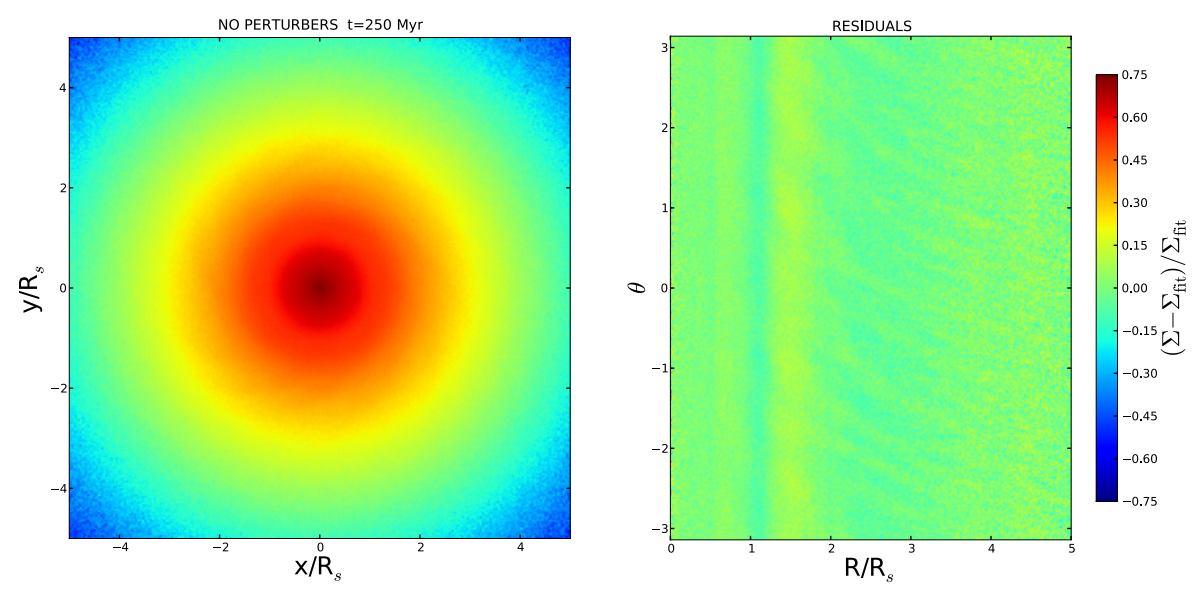

Figure 1. The outcome of an N-body experiment where a live disk of 100 million stars embedded in a dark Milky Way-sized halo is run unperturbed in isolation, after 250 Myrs. Right Panel. The residuals of the stellar surface density are shown.

matter substructures orbiting in the inner regions of galaxy halos would be destroyed by dynamical processes such as disk shocking, and hence would not be able to seed the formation of spiral structure (D'Onghia et al. 2010).

Instead, we explore the dynamical response of a disk to density inhomogeneities orbiting in the disk itself and we show that non-linear effects introduce multiple responses of the disk that affect the longevity of the resulting arms. We find that the features that are produced are long-lived and are able to self-sustain even if the perturbations driving the patterns are removed. This new and unexpected result is not predicted by standard swing amplification theory. Instead, we find that non-linear effects, not incorporated into the original Julian \& Toomre (1966), enable the patterns to survive in this regime.

The galaxy in our study consists of a Milky Way-sized dark matter halo and a rotationally supported disk of stars. The total mass of the halo is $9.5 \times 10^{11} \mathrm{~h}^{-1} \mathrm{M}_{\odot}$ computed at a radius of $160 \mathrm{kpc}$. The stellar disk follows an exponential profile and consists of 100 million particles.

\section{RESULTS}

First, we show in Figure 1 the outcome of an N-body experiment when the live stellar disk is embedded in a rigid dark Milky Way-sized halo potential and run in isolation without perturbers. After several orbital period our fiducial disk is stable and does not develop any pattern or features. The lack of features is confirmed from the Fourier component analysis in the left panel of Figure 1.

Next, we show a case where the live disk rotates with the addition of 1000 softened particles (with mass of the order of typical giant molecular clouds, $9.5 \times 10^{5} \mathrm{M}_{\odot}$ ) which are distributed within the disk and assumed to be corotating on circular orbits with the disk (see Figure 2). The live disk is shown in all cases after 250 Myrs and is displayed face-on. When the disk is perturbed by the presence of inhomogeneities co-rotating within the stellar disk on circular orbits, the disk dynamically responds to the presence of these "irritators" by developing a number of segments which resemble multi-armed structures, as shown in Figure 2. The amplitude of the Fourier components as a function of the radius in Figure 3 confirm the visual impression and indicate that the number of arms after one orbital period is approximately 7 or 8 (at two disk scale lengths). The right panel of Figure 3 shows that if the perturbers instead counter-rotate with respect the disk, there is no significant dynamical response. 

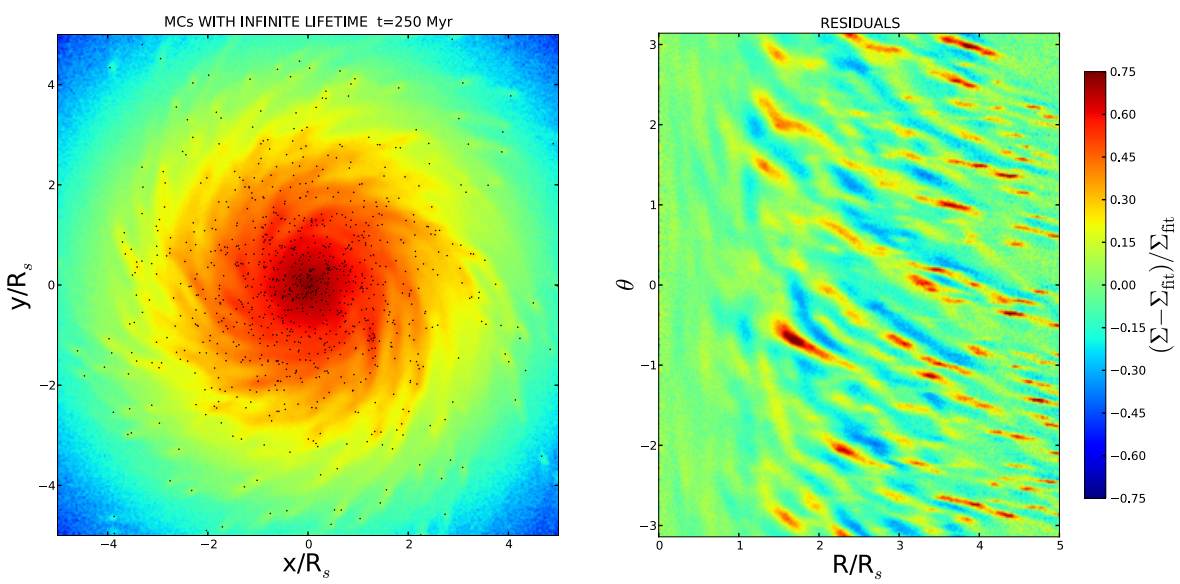

Figure 2. Left Panel. A live disk of 100 million stars embedded in a dark Milky Way-sized halo run in isolation with 1,000 softened particles (with mass of the order of typical giant molecular clouds, $9.5 \times 10^{5} \mathrm{M}_{\odot}$ ) distributed within the disk and assumed to be corotating on circular orbits within the disk. Right Panel. The residuals are shown.
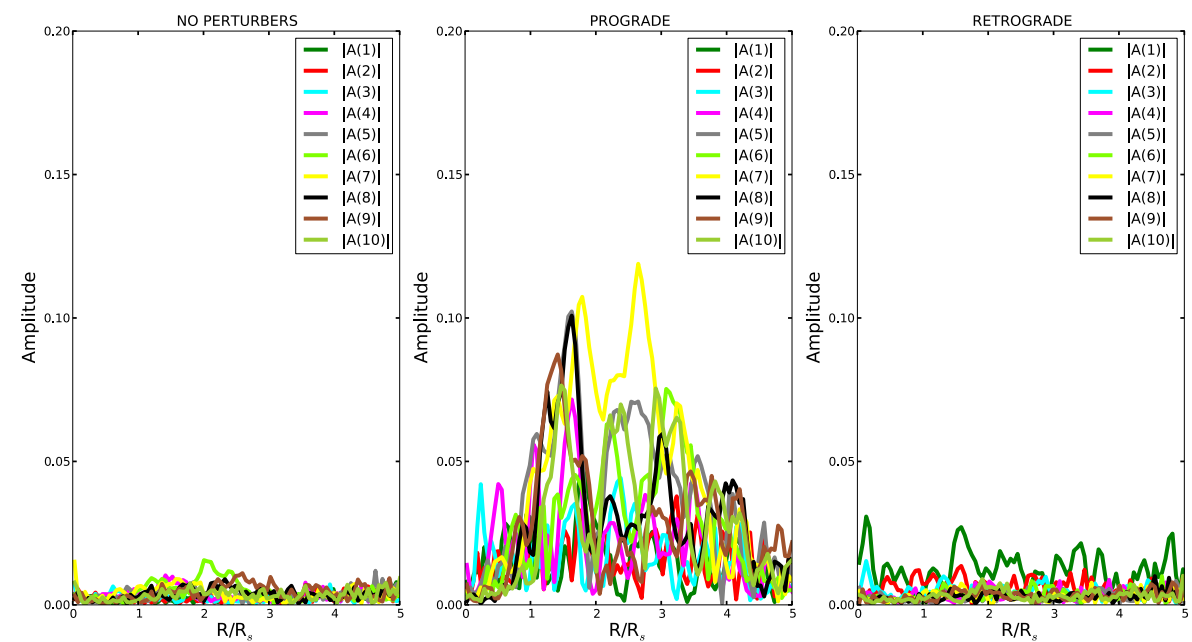

Figure 3. The amplitude of the Fourier components as a function of the radius after approximately one orbital period (250 Myrs) for three cases: an unperturbed disk (left panel), the disk perturbed by rotating inhomogeneities (middle panel) and the one with counter-rotating perturbers (right panel).

\section{DISK FRACTION AND NUMBER OF ARMS}

In the following experiments we varied the contribution of the disk to the total mass the galaxy. We keep the mass of the dark halo as in the fiducial case (Figure 1-2) but we run experiments where we increase the disk mass to the total mass of the galaxy (dark matter plus stars) from a disk fraction of $20 \%$ to the following values: $30 \%, 40 \%$ and $50 \%$ at two scale lengths of the disk. First we run these models without perturbers orbiting within the disk and verify that the disks do not develop patterns. We introduced subsequently in the initial conditions the presence of 1000 overdensities corotating with the disk. 

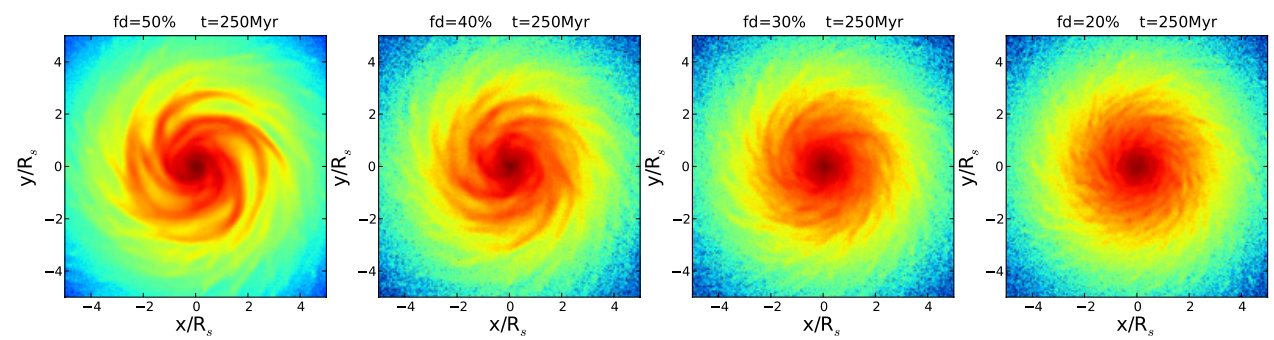

Figure 4. Disk galaxies displayed face-on for four different models. From the right to the left the contribution of the disk mass to the total mass distribution in the rotation curve of the galaxy measured at two scale lengths is increased from disk fraction of $20 \%$ (last right panel) to $50 \%$ at the left panel.

\section{CONCLUSION}

We have examined the global dynamical response of self-gravitating disks to local perturbations from overdensities. From our N-body experiments we find that a stochastic distribution of overdensities such as giant molecular clouds orbiting within the disk of stars can generate spiral pattern that extends up to 3-4 disk scale-lengths due to the swing amplification process. This process is triggered locally in the disk by the gravity exerted from the giant molecular cloud (the perturber) which evokes the formation of a wakelet in the surrounding medium that shears past it in a differential rotating disk and is amplified by the self-gravity of the stellar disk. However the arms formed in our simulations are not transients and do not fade away in a orbital period if the perturbers are removed, as predicted by the Julian \& Toomre (1966) theory. The patterns appear to be long-lived mainly due to non-linear effects, although they develop and self-maintain differently from the classical spiral density wave theory of Lin \& Shu (1964). The properties and the longevity of the arms are currently under investigation and will be the subject of a forthcoming paper.

\section{References}

[1] D’Onghia, E. et al. ApJ, 709, (2010) 1138

[2] Gauthier, J-R. et al. ApJ, 653, (2006) 1180

[3] Julian, W. H., Toomre, A. ApJ, 146, (1966) 810

[4] Lin, C. C., Shu, F. H., ApJ, 140, (1964) 646 